\title{
A Convergence Algorithm of Boundary Elements for the Laplace Operator's Dirichlet Eigenvalue Problem
}

\author{
Ali Naji Shaker \\ Directorate of Scholarships and Cultural Relations, Ministry of Higher Education and Scientific Research of Iraq, Iraq
}

Received April 7, 2021; Revised July 5, 2021;Accepted July 20, 2021

\section{Cite This Paper in the following Citation Styles}

(a): [1] Ali Naji Shaker, "A Convergence Algorithm of Boundary Elements for the Laplace Operator's Dirichlet Eigenvalue Problem," Mathematics and Statistics, Vol. 9, No. 4, pp. 579 - 587, 2021. DOI: 10.13189/ms.2021.090417.

(b): Ali Naji Shaker (2021). A Convergence Algorithm of Boundary Elements for the Laplace Operator's Dirichlet Eigenvalue Problem. Mathematics and Statistics, 9(4), 579 - 587. DOI: 10.13189/ms.2021.090417.

Copyright $(2021$ by authors, all rights reserved. Authors agree that this article remains permanently open access under the terms of the Creative Commons Attribution License 4.0 International License

\begin{abstract}
A partial differential equation has been using the various boundary elements techniques for getting the solution to eigenvalue problem. A number of mathematical concepts were enlightened in this paper in relation with eigenvalue problem. Initially, we studied the basic approaches such as Dirichlet distribution, Dirichlet process and the Model of mixed Dirichlet. Four different eigenvalue problems were summarized, viz. Dirichlet eigenvalue problems, Neumann eigenvalue problems, Mixed Dirichlet-Neumann eigenvalue problem and periodic eigenvalue problem. Dirichlet eigenvalue problem was analyzed briefly for three different cases of value of $\lambda$. We put the result for multinomial as its prior is Dirichlet distribution. The result of eigenvalues for the ordinary differential equation was extrapolated. The Basic mathematics was also performed for $\lambda$ calculations which follow iterative method.
\end{abstract}

Keywords Dirichlet Distribution, Eigenvalue Problem, Dirichlet Eigenvalue Problem, Neumann Eigenvalue Problem, Periodic Eigenvalue Problem

\section{Introduction}

Holomorphic function was investigated with the help of complex analysis, which dealt with branch of the mathematics. The function from the complex region or plane having the complex values falls in the category of holomorphic function. Differentiation of complex terms is more difficult than the real or normal differentiation. In some cases, the function is defined in a set of complex or real values termed as bounded. The Integral equation for the boundary along with its numerical approximation is popular in variety fields for eg. Insert is after wave. Helmholtz equation gives the model of scattering phenomena having appropriate conditions in presence of scattered field. There is different reformulations are observed in form of integral equation on scattering object surface.

Boundary element method comes in picture for solving the partial differential equations. This method is termed as numerical computational technique or method. This method uses the integral equation which is obtained by formulation of linear partial differential equation which represents boundary integral form. This method deals with the electromagnetic systems, acoustic systems, fluid, contact and fracture mechanics [1]. Similar to boundary element method, finite element method is used to solve issues in mathematical engineering and various mathematical models. Typical areas of working for this are mass transport, structural analysis, flow of fluid, transfer of heat etc. Basically, it is numerical method used to solve partial differential equation. It uses the 2-3 space variables. The concept of Subdivision of large system into the smaller and simple parts (system) is follow by FEM and these small parts are termed as finite elements. Algebraic equation is the outcome of finite element method. With this method, solution is occurred with minimum error function.

Boundary element as well as finite element techniques reveals some complementary properties. These properties are useful in coupling of discretization method in domain 
decomposition structure. Triangulation of boundary for computational domain and volume mesh are the requirement of the boundary element and finite element method respectively. Boundary element method is used to handle unbounded domain. Few modificaitons are necessary in finite element method for handling unbounded domain. In short, singularity approximation in boundary element method is easier than finite element. Boundary element technique is most effectual method in case of numerical problems such as adhesive contact simulation $[2,3,4]$.

In this paper, various aspects of eigenvalue problem like Dirichlet Distribution, Dirichlet Process, Dirichlet Process Mixture as a part of basics were evaluated. Dirichlet, Neumann and mixed (Dirichlet-Neumann) eigenvalue problem is discussed in detail along with the laplacian operator. Here, various scenarios related to Dirichlet eigenvalue problem was analyzed with the mathematical equations. Also, some examples were explained on the particular topic.

\section{Materials and Methods}

\subsection{Dirichlet Distribution}

The Dirichlet distribution $\operatorname{Dir}(\alpha)$ is a family of continuous multivariate probability distributions parameterized by a vector $\alpha$ of positive reals. Following equation represents the Dirichlet Distribution

$$
p\left(P=\left\{p_{i}\right\} \mid \alpha_{i}\right)=\frac{\prod_{i} \Gamma\left(a_{i}\right)}{\Gamma\left(\sum_{i} a_{i}\right)} \prod_{i} p_{i}^{a_{i-1}}
$$

Here, the value of $p_{i}$ is greater than 1 and the sum of this term for its value is equal to 1 .

There are two parameters: concentration of scaling parameter, i.e. $\sigma$ which is termed as $\sum_{i} a_{i}$ and base measure which is represented by $\frac{a_{i}}{\sigma}$.

As Beta generalization is explained with two aspects: beta becomes distribution over bionomial and Dirichlet is the distribution over the multinomial which is simple as discussed above with reference to above equation (1). Dirichlet is the base to the multinomial means Dirichlet come first, then multinomial distribution $[5,7,8]$.

Assume that every experiment is resulted in the ' $\mathrm{j}$ ' possible outcomes having probabilities $p_{1}, p_{2}, \ldots \ldots \ldots \ldots, p_{j}$. Multinomial mockup the distribution of vectors which shows that $\mathrm{N}$ trial, how many times, observation of every outcome is done. Mathematically, it is represented as

$P\left(a_{1}, a_{2}, a_{3}, \ldots \ldots, a_{j} \mid n, p_{1}, p_{2}, p_{3}, \ldots . p_{j}\right)=\frac{N !}{\prod_{i=1}^{j} a_{i} !} p_{i}{ }^{a}$

\section{And}

$N$ with $x_{i}$ is greater than or equal to 0

$\sum_{i} x_{i}=$

Figure 1 represents the multinomial distributions for different probabilities.

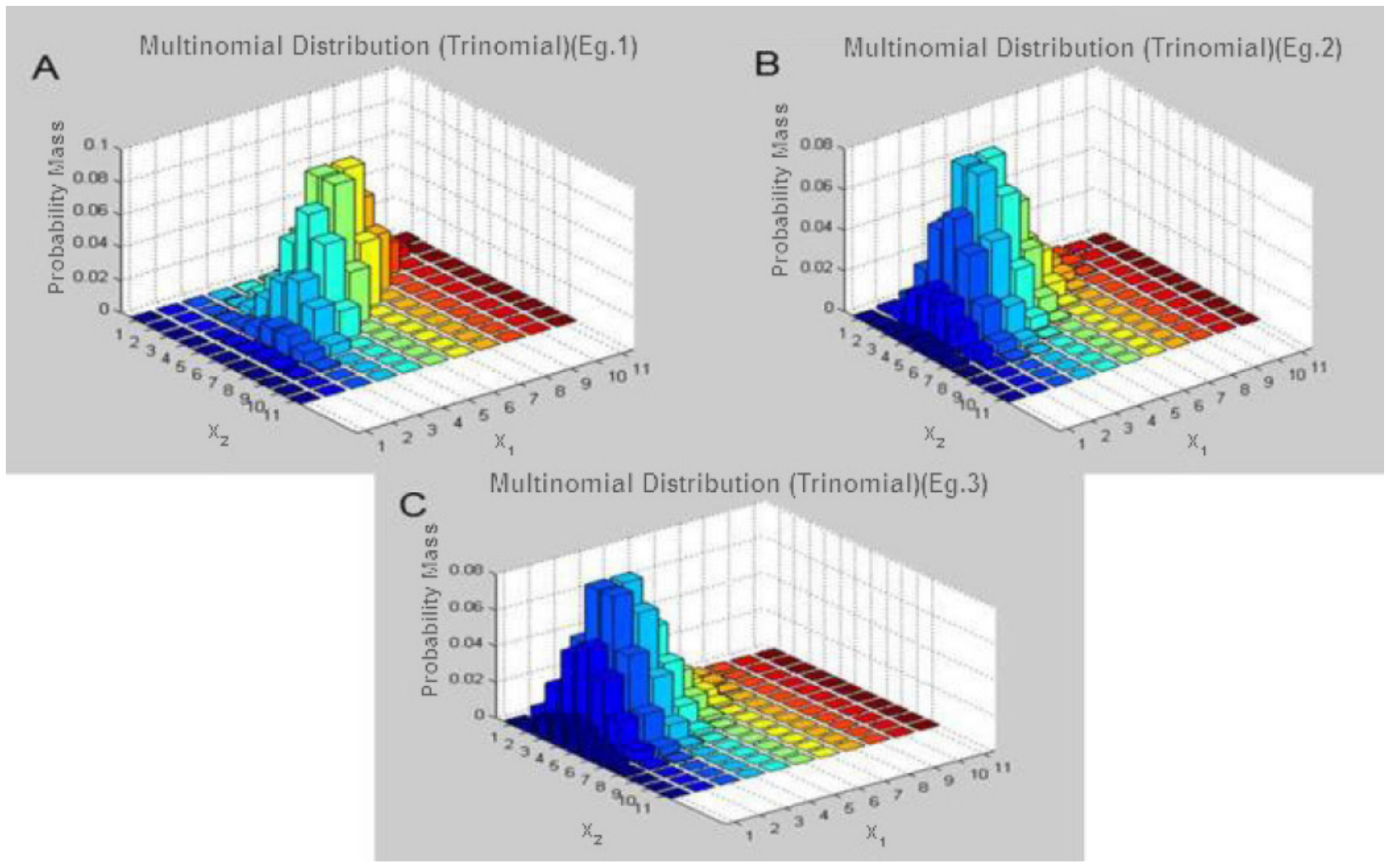

A: Graph for Probability 1/2 1/3 1/6; B: Graph for probability 1/3 1/4 5/12; C: Graph for probability 1/4 1/4 1/2

Figure 1. Probability Graph for $1 / 2 \quad 1 / 31 / 6$ 
Generally Dirichlet theorem states that any function say $\mathrm{f}(\mathrm{x})$ with interval $[-n,+n]$ is able to expand with the help of fourier series. The euqation for the fouries series is

$$
f(x)=a_{0}+\sum_{n=1}^{\infty}\left(a_{n} \sin \frac{n \pi x}{a}+b_{n} \cos \frac{n \pi x}{a}\right)
$$

Dirichlet theorem deal with prime numbers and plays a vital role in case of various solutions [5].

\subsection{Dirichlet Process}

Dirichlet process is a probability distribution whose range is itself a set of probability distributions. Eg. Many different colours balls are present in box. Hence, the probability of choosing and picking the new color ball goes on decreasing. The proportion of the balls in box after an infinite withdraws is the dirichlet process. Generally, the Dirichlet represents the probability of the next symbol. Dirichlet distribution becomes simple with Dirichlet process. The few examples based on Dirichlet process are poly urn method, process of Chinese restaurant, stick breaking techniques and construction of gamma process.

The concept of Dirichlet process can be understood easily with an example. Figure 2 shows the sitting arrangement. The possibility of individual join the table and grouping concept can be evaluated. Figure 2 shows the probability scenario in presence of 5 and 6 customer.

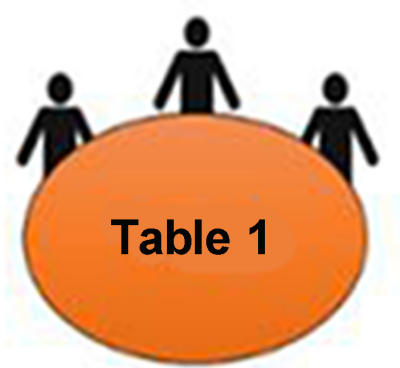

$P$ (Customer 5 sit at table 1)

$$
=\frac{3}{a+4}
$$

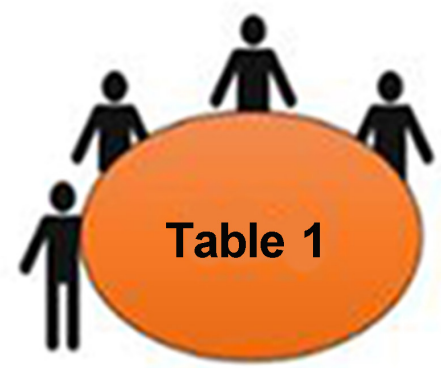

$P$ (Customer 6 sit at table 1)

$$
=\frac{4}{a+5}
$$

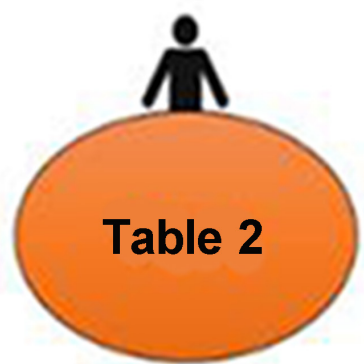

$P$ (Customer 5 sit at table 1)

$$
=\frac{1}{a+4}
$$

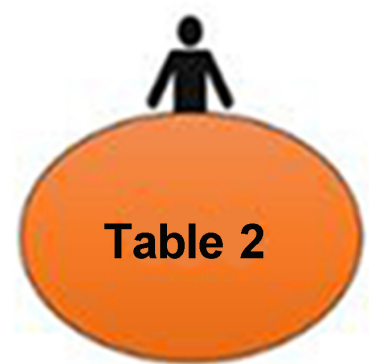

$P($ Customer 6 sit at table 1)

$$
=\frac{1}{a+5}
$$
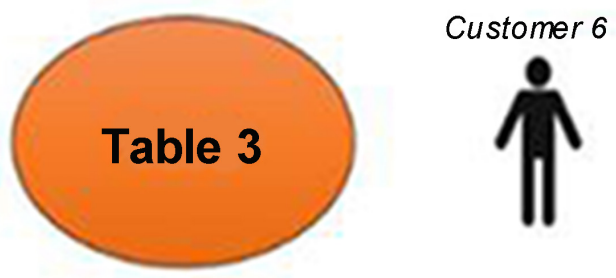

Customer 5
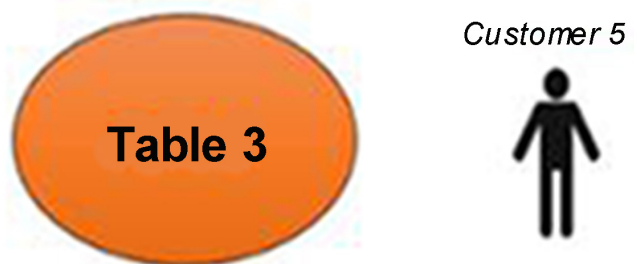

$P$ (Customer 5 sit at table 3)

$$
=\frac{a}{a+4}
$$

$$
=\frac{a}{a+5}
$$

Figure 2. Dirichlet distribution 


\subsection{Dirichlet Process Mixture}

It is the simple concept which can be elaborated in the below Figure 3 .

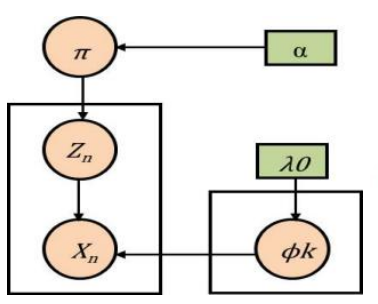

$\mathbf{A}$
B

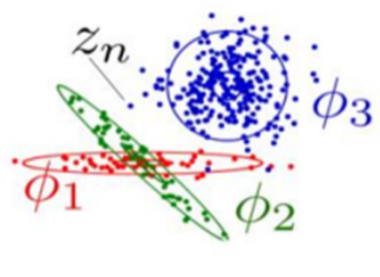

Figure 3. Dirichlet mixture model

Dirichlet distribution is popular as it is a prime distribution for the multinomial parameters since Dirichlet becomes conjugate for multinomial under assumption of some parameters. It has some limitations such as every variable have own mean with common variance and second its variable gets added in presence of mutual independency between them. These limitations are overcome by keeping computational simplicity having conjugate distribution to multinomial with different parameters. Hence, the tree structure is comes in picture and was well explained by [9].

\subsection{Eigenvalue Problem}

Some of the examples based on the heat equations require the transformation from partial differential equations to ordinarily differential equation. The ordinary differential equation is obtained in form of eigenvalue problem. We generate the result for ordinary differential equation with graphical representation. There are various eigenvalue problems: First eigenvalue problem (Dirichlet Eigenvalue problem), Second Eigenvalue problem (Neumann Eigenvalue problem), third eigenvalue problem (Mixed Dirichlet-Neumann Eigen value problem) and forth eigenvalue problem (periodic eigenvalue problem). In this paper we are going to study Dirichlet eigenvalue problem. Shortly mathematical equations of other eigenvalue problem are discussed $[10,11]$.

\subsubsection{First Eigenvalue Problem}

It is also known as Dirichlet Eigenvalue problem. For discussion of this point we have to use second order differential equation.

$$
\frac{d^{2} X}{d x^{2}}+\lambda X=0 \quad, 0<y<m
$$

Having the Dirichlet boundary condition

$$
X(0)=0 \quad \text { and } X(m)=0
$$

We aimed to find out the value of the $\lambda$ with non-trivial solution of above equation (4). Conditions assumed for this problem are homogeneous and linear
[12]. Hence, the nonzero output is the solution to that problem and it is same as to the $X(x)$. This is known as the eigenvalue problem.

The Dirichlet Eigenvalue problem includes, determining of solution $X(x)$ which has $[0, \mathrm{~m}]$ domain where the few values of $\lambda$ will satishfied boundary conditon, $X(0)=X(m)=0$. So we have three cases where possible solutions are falling, i.e. $\lambda=0, \lambda<$ 0 and $\lambda>0$.

Case 1: $\lambda=\mathbf{0}$

$$
\begin{aligned}
\frac{d^{2} X}{d x^{2}}+\lambda X & =0 \\
X^{\prime \prime}+\lambda X & =0
\end{aligned}
$$

Put the value of $\lambda=0$ then the equation becomes

$\therefore$ we get,

$$
X^{\prime \prime}=0
$$

General solution of equation (5) is

$$
X(x)=c_{1}+c_{2} x
$$

where $c_{1}$ and $c_{2}=$ Constants.

For fullfilling the boundary condition $\mathrm{X}(0)$,it is must to have $c_{1}=0$.

Hence equation (6) becomes

$$
X(x)=c_{2} x
$$

On the same sense, for the fulfillment of the next boundary condition $\mathrm{X}(\mathrm{m})=0, c_{2}$ should be 0 .Hence, finally we get the solution $X(x)=0$ for $\lambda=0$ and so $\lambda=0$ not eigenvalue.

\section{Case 2: $\lambda<0$}

As, $\lambda<0$. So, after considering $\lambda=-\omega^{2}$ the equation (4) becomes

$$
X^{\prime \prime}-\omega^{2} X=0
$$

General solution for that equation is

$$
X(x)=c_{1} e^{\omega x}+c_{2} e^{-\omega x}
$$

where $\quad c_{1}$ and $c_{2}=$ Constants.

The $X(x)$ boundary condition requires the $c_{2}=-c_{1}$. The eq. (8) becomes

$$
\begin{gathered}
X(x)=c_{1} e^{\omega x}-c_{1} e^{-\omega x} \\
X(x)=c_{1}\left(e^{\omega x}-e^{-\omega x}\right) \\
X(x)=2 c_{1} \sinh \omega x
\end{gathered}
$$

Since the another boundary condition $\mathrm{X}(\mathrm{m})=0$ needed $2 c_{1} \sinh \omega x=0$,it means $c_{1}=0$. From all of mathematical equations it is observed that eigenvalue problem has trivial solution $(X(x)=0)$ for $\lambda<0$. So we conclude that values of $\lambda$ less than 0 are not an eigenvalues. 


\section{Case 3: $\lambda>0$}

As $\lambda>0$. So, after placing $\lambda=\omega^{2}$ the equation (4) becomes

$$
X^{\prime \prime}+\omega^{2} X=0
$$

General solution for that equation is

$$
X(x)=c_{1} \cos \omega x+c_{2} \sin \omega x
$$

where $c_{1}$ and $c_{2}=$ Constants.

For satisfying the boundary condition $X(x)=0$, it must be that $c_{1}=0$.

Hence, eq(11) becomes

$$
X(x)=c_{2} \sin \omega x
$$

But for filling the another boundary condition $X(m)=0, c_{2} \sin \omega m=0$

From above it is observed that $c_{2}=0$ gives the trival solution for equation (4). For obtaining non-trivial solution, $\omega$ must have positive root of equation

$$
\sin \omega m=0
$$

After solving above equation we will get,

$$
\begin{gathered}
\omega m=\sin ^{-1}(0) \\
\omega m=\pi \\
\omega=\frac{n \pi}{m} \quad \text { for } n=1,2,3,
\end{gathered}
$$

And exclude for $c_{2}$, we get

$$
X(x)=\sin \frac{n \pi}{m} x \quad \text { for } n=1,2,3,
$$

For the different values of $n=1,2,3, \ldots \ldots \ldots, \lambda$ is obtained as

$$
\begin{gathered}
\lambda=\omega^{2}=\left(\frac{n \pi}{m}\right)^{2} \\
\lambda_{n}=\left(\frac{n \pi}{m}\right)^{2}
\end{gathered}
$$

From this third case, it is observed that the equation (4) have non-trivial solution and these are the eigenvalues for that problem. Finally, we get the eigen function as below

$$
X_{n}(x)=\sin \frac{n \pi x}{m} \quad \text { for } n=1,2,3,
$$

\subsubsection{Second Eigenvalue Problem (Neumann Eigenvalue Problem)}

For the boundary condition $X^{\prime}(0)=X^{\prime}(m)=0$, we have the eigenfunction for Neumann eigenvalue problem stated as below

$$
X_{n}(x)=\cos \frac{n \pi x}{m} \quad \text { for } n=1,2,3,
$$

\subsubsection{Third Eigenvalue Problem (Mixed}

Dirichlet-Neumann Eigenvalue Problem I )

For the boundary condition $X(0)=X^{\prime}(m)=0$, we have the eigenfunction formixed Dirichlet-Neumann eigenvalue problem

$$
X_{n}(x)=\sin \frac{\left(n-\frac{1}{2}\right) \pi x}{m} \quad \text { for } n=1,2,3
$$

\subsubsection{Third Eigenvalue Problem (Mixed}

Dirichlet-Neumann Eigenvalue Problem II)

For the boundary condition $X^{\prime}(0)=X(m)=0$, we have the eigenfunction for mixed Dirichlet-Neumann eigenvalue problem

$$
X_{n}(x)=\cos \frac{\left(n-\frac{1}{2}\right) \pi x}{m} \quad \text { for } n=1,2,3
$$

2.4.5. Forth Eigenvalue Problem (Periodic Eigenvalue Problem )

For the boundary condition $X(0)=X(m)$ and $X^{\prime}(0)=X^{\prime}(m)$, we have the eigenfunction forperiodic eigenvalue problem

for $X_{0}, X_{n}(x)=\cos \frac{2 n \pi x}{m}, \sin \frac{2 n \pi x}{m} \quad$ for $n=1,2,3$,

From the above eigenvalue problem equation it is observed that, somehow it is similar to fourier series. Hence, previously we mentioned that Dirichlet theorem states that any function is expandable with fourier series.

\subsection{Eigenvalues with Laplacian Operator}

Consider the internal Dirichlet eigenvalue problem of Laplace operator

$-\Delta u(p)=\lambda u(p) \quad$ where $p \in \Omega, u(p)=\underset{\Gamma}{0}$ where $p \in$
$\Gamma=\partial \Omega$

$\Omega \subset \mathbb{R}^{3}=$ lipschitz domain which is Bounded

Transformation of above eigenvalue problem into its analogous nonlinear eigenvalue problem is given [5].

$$
\frac{1}{4 \pi} \int_{\Gamma} \frac{\cos (\kappa|p-q|)}{|p-q|} t(q) d s_{q}=0 \text { for } p \in \Gamma=\partial \Omega
$$

Where, $\kappa=\sqrt{\lambda}, t(q)=m_{y} . \nabla u(q)$ for the $q \in \Gamma$.

Related Eigen function for equation 1 is shown belows equation 25 .

$$
u(p)=\frac{1}{4 \pi} \int_{\Gamma} \frac{\cos (\kappa|p-q|)}{|p-q|} t(q) d s_{q}=0 \text { for } p \in \Omega
$$

Equivalent boundary element scenario for eignevalue problem for equation 1 was summarized by various authors [13, 14]. Non-linear eigenvalue problem with polynomial approximation (Approximation of boundary element) was discussed by [15]. Exact solutions for Laplace equation with method of differential transform was discussed by Jamil et al. [4], Existence along with Uniqueness of Solutions by the $\mathrm{p}(\mathrm{x})$-Laplacian Equation (Convection) was derived by Wang et al, [16] and generalization of the laplacian differential operator was well explained by Romero, [17].

Here we considered the concept of iterative solution is considered for nonlinear eigenvalue problem which is 
similar to inverse iteration for matrix of nonlinear as well as linear eigenvalue problem. Newton method is applied to equation 2 for getting solution of nonlinear equation. In that case, proper scalping condition is introduced at the time of addition. Theoretically, there is occurrence of simple eigenvalues. Galerkin boundary element technique is also used for the getting solution of nonlinear eigenvalue problem. Detailed numerical analysis is given in $[18,19,20,21]$. Further, mathematical derivation was related to boundary element method.

Following equation represents the nonlinear eigenvalue problem

$$
\left(W_{\kappa} t\right)(p)=\frac{1}{4 \pi} \int_{\Gamma} \frac{\cos (\kappa|p-q|)}{|p-q|} t(q) d s_{q}=0 \text { for } p \in \Gamma
$$

Where, operator $W_{\kappa}: H^{-1 / 2}(\Gamma) \rightarrow H^{1 / 2}(\Gamma)$, is bounded and linear for fixed value of $\kappa$.Scaling condition is introduced with the help of similar standard in $H^{-1 / 2}(\Gamma)$ for normalizing eigen solution $t \in H^{-1 / 2}(\Gamma)$ for equation 26.

$$
\|t\|_{W}^{2}=\left\langle W_{t, t}\right\rangle_{\Gamma}=\frac{1}{4 \pi} \int_{\Gamma} t(p) \int_{\Gamma} \frac{1}{|p-q|} t(q) d s_{q} d s_{p}=1
$$

Where

$W: H^{-1 / 2}(\Gamma) \rightarrow H^{1 / 2}(\Gamma)=$ single layer potential of laplace operator.

Let's focus on nonlinear eigenvalue problem solutions $(t, \kappa) \in H^{-1 / 2}(\Gamma) \times \mathbb{R}$.

$$
\begin{gathered}
F_{1}(t, \kappa)=\left(W_{k} t\right)(p)=0, F_{2}(t, \kappa)=\left(W_{t, t}\right)_{\Gamma}-1= \\
0 \text { for } p \in \Gamma
\end{gathered}
$$

Hence the function $\mathrm{F}$ is $H^{-1 / 2}(\Gamma) \times \mathbb{R} \rightarrow H^{1 / 2}(\Gamma) \times \mathbb{R}$ defined as

$$
F(t, \kappa)=\left(\begin{array}{l}
F_{1}(t, \kappa) \\
F_{2}(t, \kappa)
\end{array}\right)=\left(\begin{array}{c}
\frac{1}{4 \pi} \int_{\Gamma} \frac{\cos (\kappa|p-q|)}{|p-q|} t(q) d s_{q} \\
\left(W_{t, t}\right)_{\Gamma}-1
\end{array}\right)
$$

To getting Eigen solution for scaled eigenvalue problem given in equation 28 , we need to search solution $(t, \kappa) \in H^{-1 / 2}(\Gamma) \times \mathbb{R}$ of nonlinear equation.

$$
F(t, \kappa)=0
$$

This is solved using Newton method. After application of the Newton method for finding solution $\left(t_{*} \kappa_{*}\right) \in$ $H^{-1 / 2}(\Gamma) \times \mathbb{R}$ of nonlinear equation given in eq.30 and $\left(t_{m+1} \kappa_{m+1}\right) \in H^{-1 / 2}(\Gamma) \times \mathbb{R}$ can be obtained with linear operator equation

$$
F^{\prime}\left(t_{m} \kappa_{m}\right)\left(\begin{array}{c}
t_{m+1-} t_{m} \\
\kappa_{m+1-} \kappa_{m}
\end{array}\right)+F\left(t_{m} \kappa_{m}\right)=0
$$

Adequate conditions are given in following theorem so equation 31 is solved and Newton technique converges into Eigen solution $\left(t_{*} \kappa_{*}\right)$.
Theorem: Consider $F(t, k)$ has solution $\left(t_{*} \kappa_{*}\right)$. Presume

$(a 1) \kappa_{*}=$ Simple eigen value of $W_{\kappa} t=0$,

(a2) $A_{\kappa^{*}} t_{*} \notin \mathcal{R}\left(W_{\kappa *}\right)$

Hence the $F^{\prime}\left(t_{*} \kappa_{*}\right)$ is reversible and newton's technique converges into initial values in adequate small neighborhood $V_{\rho}\left(t_{*} \kappa_{*}\right)$ to $\left(t_{*} \kappa_{*}\right)$ with $>0$.

$$
\left\|t_{*}-t_{m}\right\|_{H^{-1 / 2}(\Gamma)}^{2}+\left|\kappa_{*}-\kappa_{m}\right|^{2} \leq \rho^{2}
$$

For number of eigen values $\kappa_{*}$, Frechet derivative is not suitable as $F^{\prime}(t, k)$ is infusable.However, Newton techniques might converge [22,23]. It may possible that convergence rate will be small and domain is large neighborhood of resultant but constricted area which keep away from set where, $F^{\prime}$ is singular. In the present study, we put the examples which show that multiple eigenvalues can be obtained by Newton's method means Newton techniques converges for many eigen values $[12,18,24,25]$.

Other method are also available for the solving the problem for laplacian operator. A meshless method was explained by Kamran et al. [26] which is based on laplace transform for the two dimensional multiple term time for fractional partial integral differential equations [26]. Same scenario with approximation of matrics and spectral analysis was done by Aceto, et al [27]. Discrete laplacian operator along with application $\mathrm{s}$ in the area of signal processing were discussed by Waheed et al. [28]. The concept of the concave and convex non linarite is for the p-laplacian equation with multiple solutions is given by chen et al. [29]. Critical fractional laplacian equation in presence of a perturbation gave bifurcation and symmetrical result. This concept was given by Zuo et al. [30].

\section{Results and Discussion}

Example 1: Solution for ordinary equation $\frac{d x}{d t}=A x$

Its solution is represented in matrix exponential form as follows

$$
x(t)=e^{t A} x(0)
$$

Here we obtain the coefficient matrix A, value of lamda $(\lambda)$, is nonsingular matrix represents eigenvalue decomposition, diagonal elements of $\mathrm{D}$ represent eigenvalues. Following figure (4) shows that the solution swirling in the direction of origin. That behavior is associated with eigenvalues of coefficient matrix. 
$\mathrm{A}=$

$$
\begin{array}{rrr}
0 & -5 & -1 \\
5 & 2 & -15 \\
-4 & 16 & -8
\end{array}
$$

lamda $=$

$$
-2.4888
$$

$-1.7556+15.2702 \mathrm{i}$

$-1.7556-15.2702 \mathrm{i}$

$\mathrm{v}=$

$$
\begin{array}{lll}
-0.8536 & -0.0688-0.2353 \mathrm{i} & -0.0688+0.2353 \mathrm{i} \\
-0.3472 & -0.7030 & -0.7030 \\
-0.3884 & -0.1989+0.6372 \mathrm{i} & -0.1989-0.6372 \mathrm{i}
\end{array}
$$$$
\mathrm{D}=
$$

$$
\begin{array}{ccc}
-2.4888 & 0 & 0 \\
0 & -1.7556+15.2702 \mathrm{i} & 0 \\
0 & 0 & -1.7556-15.2702 \mathrm{i}
\end{array}
$$

\section{Example 2: Calculation of $\lambda$}

Here, we discuss investigation of behavior of nonlinear boundary element along with few numerical outcome. We assumed the internal Dirichlet eigenvalue problem represented by equation 1 . It has the domain $[0, \mathrm{~m}]=$ $\left(0, \frac{1}{2}\right)^{3}$ is cube. So, eigen values stated as

$$
\lambda_{k}=4 \pi^{2}\left[k_{1}^{2}+k_{2}^{2}+k_{3}^{2}\right]
$$

Its related eigen function is shown in eq. 12

$$
u_{\kappa}(p)=\left(\sin 2 \pi k_{1} p_{1}\right)\left(\sin 2 \pi k_{2} p_{2}\right)\left(\sin 2 \pi k_{3} p_{3}\right)
$$

First eigenvalue for $k_{1}=k_{2}=k_{3}=1$

$$
\begin{gathered}
\lambda_{1}=4 \pi^{2}\left[1^{2}+1^{2}+1^{2}\right] \\
\lambda_{1}=4 \pi^{2}[3] \\
\lambda_{1}=12 \pi^{2} \\
\kappa_{1}=\sqrt{12 \pi^{2}}=\sqrt{4 \times 3 \pi^{2}}=2 \sqrt{3} \pi=10.88279
\end{gathered}
$$

Similarly, second eigenvalue for $k_{1}=2, k_{2}=k_{3}=$ 1

$$
\begin{gathered}
\lambda_{2}=4 \pi^{2}\left[2^{2}+1^{2}+1^{2}\right] \\
\lambda_{2}=4 \pi^{2}[6] \\
\lambda_{2}=24 \pi^{2} \\
\kappa_{2}=\sqrt{24 \pi^{2}}=\sqrt{4 \times 6 \pi^{2}}=2 \sqrt{6} \pi=15.39
\end{gathered}
$$

Here, we obtained the value of $\lambda$ greater than 0 . Hence, we get the non-trivial solution to that equation with well-defined eigenvalue function for different values of $n$ in a given domain.

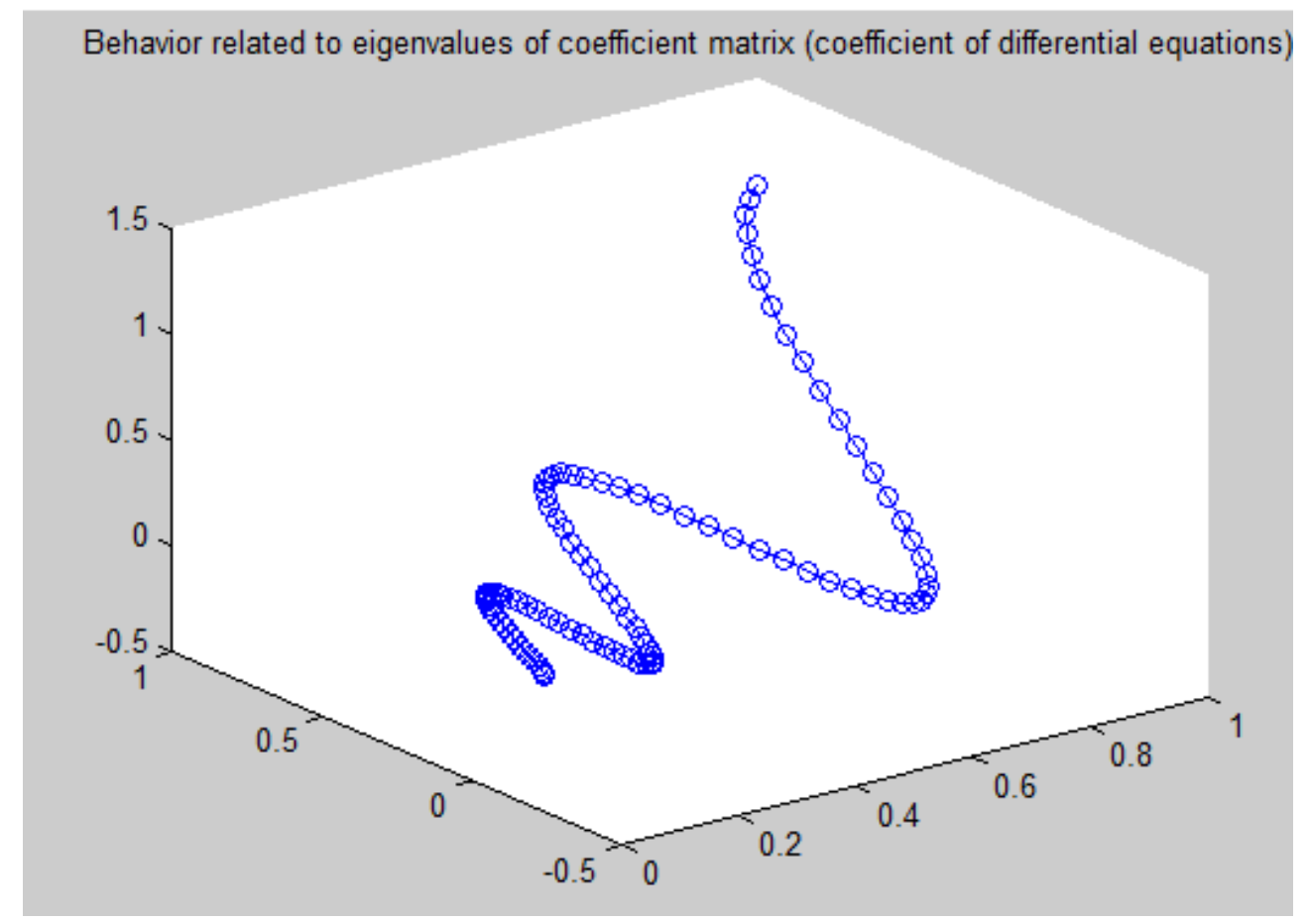

Figure 4. Behaviour of eigenvalue of coefficient matrix 


\section{Conclusions}

This paper is focused on the Dirichlet eigenvalue problem. With reference to Dirichlet eigenvalue problem we discussed Dirichlet distribution, Dirichlet process, Model of mixed Dirichlet distribution. Various eigenvalue problems were summarized in this paper along with eigen function for them. Results were obtained for the multinoial distribution for different probabiltiy values, solution of ordinary differential equation with behavior of eigenvalue of coefficient matrix. From above mathematical analysis (in case of Dirichlet eigenvalue problem), it is concluded that the non-trivial solution is obtained only when $\lambda$ has value greater than 0 .

As per author knowledge, very few articles were published on the boundary conditions in the presence of dirichlet eigen value problem. Hence, rarely a few algorithms are available in this area. The author suggests that, it is a great chance to upcoming researchers to develop algorithms with lower complexity in terms computation and also allow to solve the problem of eigen values for various values of $\lambda$ considering $\lambda$ has value less than 0 .

\section{REFERENCES}

[1] S Kirkup. The boundary element method in acoustics: A survey. Applied Sciences; 9, 1642, 1-48, 2019.

[2] U. Langer, Institute For Computational Mathematics, University of Johannes Kepler, Austria. 2019

[3] E Akhmetgaliyev, O Bruno and N Nigam., A Boundary integral algorithm for the laplace Dirichlet- Neumann mixed eigenvalue problem. Computing \& Mathematical Sciences, University of Simon Fraser, 1-43, 2014

[4] M Jamil, M Yaseen, Rabia I. Exact solutions of Laplace equation by differential transform method arXiv:1312.7277vl [math.AP] 2013

[5] Dilan G, Carl R. Dirichlet Process Gaussian Mixture Models: Choice of the Base Distribution. Journal of Computer Science and Technology 25(4):653-664, 2010

[6] E V Mayamol. Some properties of a generalized type-1 dirichlet distribution. Statistica, Anno Lxx.;1,1- 17, 2010.

[7] T P Minka. Estimating a dirichlet distribution. Microsoft Research Paper 2012;1-17.

[8] M I Jordan. Dirichlet distribution and dirichlet process. Stat 260: Bayesian Modeling And Inference. 1-5, 2010

[9] T Minka. The Dirichlet-Tree Distribution. Microsoft Research Paper, 1-6, 2004

[10] A Salih. Eigenvalue Problem. Department of Aerospace Engineering Indian Institute of Space Science and Technology, Thiruvananthapuram, 1-12, 2016

[11] H.M Fahad, M. Rehman, A. Fernandez. On Laplace transforms with respect to functions and their applications to fractional differential equations. arXiv:1907.04541v2 [math.CA] 2020

[12] X Fan, Q Zhang, D Zhao. Eigenvalues of P(X)-Laplacian dirichlet problem. Journal of Mathematical Analysis and Applications, 302, 306-317, 2003

[13] N Kamiya, E Andoh; K Nogae. Eigenvalue analysis by the boundary element method: new developments. Engng. Anal. Bound. Elms.;12,151-162, 1993

[14] S M Kirkup; S Amini. Solution of the helmholtz eigenvalue problem via the boundary element method. Internat. J. Numer. Methods Engrg. 36,321-330, 1993

[15] DW Decker, CT Kelley. Convergence acceleration for newton's method at singular points. SIAM Journal on Numerical Analysis, 19(1), 219-229, 1982

[16] B Wang, G Hou, B Ge. Existence and uniqueness of solutions for the $\mathrm{p}(\mathrm{x})$-laplacian equation with convection term Mathematics, 8(10), 1768, 2020

[17] Romero, L.G. A generalization of the Laplacian differential operator. Palestine Journal of Mathematics 5(2), 204-207, 2016

[18] Steinbach and G Unger. A boundary element method for the dirichlet eigenvalue problem of the laplace operator. Berichte Aus Dem Institut F"Ur Numerische Mathematik, Tu Graz, Bericht., 1-5. 2007

[19] V Mehrmann and H Voss. Nonlinear eigenvalue problems: a challenge for modern eigenvalue methods. Gamm Mitt. Ges. Angew. Math. Mech.; 27(2), 121-152, 2005.

[20] A Ruhe. Algorithms for the nonlinear eigenvalue problem. SIAM Journal on Numerical Analysis; 10, 674-689, 1973

[21] JH Wilkinson., The algebraic eigenvalue problem. Clarendon Press. Oxford. 1965

[22] DW Decker and CT Kelley. Newton's Method at Singular Points. I. SIAM Journal on Numerical Analysis; 17(1), $66-70,1980$

[23] DW Decker, CT Kelley. Convergence acceleration for newton's method at singular points. SIAM Journal on Numerical Analysis, 19(1), 219-229, 1982

[24] ILy. The first eigenvalue for the P-Laplacian operator. J. of Inequalities In Pure and Applied Mathematics.; 6(3), 1-12, 2005.

[25] B Bogosel, A Henrot, I Lucardesi. Minimization of the eigenvalues of the dirichlet-laplacian with a diameter constraint. SIAM Journal on Mathematical Analysis, 2018

[26] K Kamran, Z Shah, P Kumam, N.A. Alreshidi. A meshless method based on the laplace transformfor the 2D multi-term time fractional partialintegro-differential equation. Mathematics, 8(11), 1972, 2020

[27] L. Aceto, M. Mazza, S Stefano. Fractional Laplace operator in two dimensions, approximating matrices, and related spectral analysis. Calcolo (2020) 57:27, 2020

[28] W. Waheed, G. Deng, B. Liu. Discrete Laplacian Operator and Its Applications in Signal Processing. IEEE Access, 8: 89692-89707, 2020. 
[29] Q. Chen, C Chen., Y Shi. Multiple solutions for fractional p-Laplace equation with concave- convex nonlinearities. Chen et al. Boundary Value Problems 2020: 63, 2020
[30] J. Zuo, M. Li, B Li., Z Qiao. A bifurcation and symmetry result for critical fractional Laplacian equations involving a perturbation. Advances in Difference Equations 2020:58, 2020 